\title{
Design of A New Non-Contact Screw Seal and Determination of Performance Characteristics
}

\author{
Vildan Girişta Kaya, Vedat Temiz, Zeynep Parlar, Levent Kavurmacıoğlu \\ Istanbul Technical University, Mechanical Engineering Faculty \\ Mechanical Engineering Department, Istanbul, Turkey \\ girista@itu.edu.tr; temizv@itu.edu.tr; parlarze@itu.edu.tr; kavurmacio@itu.edu.tr
}

\begin{abstract}
Non-contact seals are optimal solutions for the applications operating with relatively high speeds. In these seals, positively displacement pump's principle is applied. One sub-category of non-contact seals, screw seals are studied in detail, including their working principles, operating limits and technical properties. In current work, two part screw geometries having right and left hand screw threads on each side is developed for analysis. Mainly, sealing performance of contactless screw seals is investigated both experimentally and numerically. For this purpose, a new test system is designed. Seal system is tested both oil-free and oil-supplied running conditions. The pressure distribution and friction torque values are recorded on the experiments. Numerical analysis are carried out regarding experimental data.
\end{abstract}

Keywords: visco-seal, screw seal, contactless sealing, active seals

\section{Introduction}

With development of the machine technology, sealing elements, play an important role in the operation of mechanical systems. Especially in high operating speed systems, contactless seals are used in order to avoid possible damage due to friction. In contactless sealing systems, there is no contact between the seal and machine parts in the sealing area. With the help of the screw profiles either on the shaft or on the hub, a narrow groove is formed and sealing is achieved by the flow resistance along this continuous groove. The flow resistance, also called as the pump effect, depends on the centrifugal forces and/or viscous flow as well as the surface geometry. In the sealing systems, in order to control the leakage flow by reducing the functional gap, active sealing methods are also available as an alternative method. In the active sealing system, leakage flow is directed back into the system with the help of the sealing element's geometry. In this narrow gap, the fluid dragged along with the moving surface, is flowed back into the system with the help of the dynamic pressure generated between the two surfaces.

In the study of Ludwig, Strom and Allen, the effect of screw profiles of the seal, to the pressure distribution was analysed. According to this study, in the plane of rotation, circumferential pressure gradients are linearly increased in the thread gap and linearly decreased on thread tip. It was determined that every screw and thread repeats the same pressure profile and has a saw-teeth character on the rotational plane [1]. In the study of Childs, leakage tests for helically grooved seals show that, with the increase of the operating speed, the amount of leakage flow decreases. The leakage flow increases; with the increase of the screw thread depth and pitch angle; decreases with the reducing of radial clearance [2]. In another study of Childs, the effect of the screw profile on the sealing performance was investigated. It was occurred that with an increase on the helix angle, leakage flow increased. In case the helix angle was higher than $30^{\circ}$, leakage was greater than for sealing elements with no screw profiles at all [3]. In the experimental and theoretical studies on screw seals by Zuk, Ludwig and Johnson, the effect of forming the screw profiles either on the shaft or housing was inspected and unformed rotor-screw profiled housing design gives higher sealing capacity [4]. Patterson examined the mathematical models developed for screw seals and customized the Hodgson model to multi-threaded screws. Based upon the basic flow equation, sealing coefficient and leakage flow expressions were obtained and an expression to achieve the pressure difference at zero leakage is developed [5].

In another study of Ludwig, Strom and Allen, in order to examine the sealing performance of the helically grooved screw seal, liquid sodium and water were used as fluid. At the unformed shaft and grooved housing combination, gas bubbles were held out of the grooves with the effect of centrifugal forces. The experiments with grooved shaft and 
unformed rotor combination showed that gas bubbles were pumped into the groves due to centrifugal effect. It was seen these two different shaft-housing combinations have different sealing performance characteristics [6]. According to a study of Boon, parameters like high helix angle, multiple thread, large tip thread width or high screw thread depth do not effect on gas ingestion in screw seal elements. Again in this study, it was concluded that, increase of gas ingestion decreased the sealing performance. At higher rotating speeds, sealing performance drop was increased due to higher gas ingestion [7].

In this study, performance characteristics of screw seal elements that operate contactlessly according to principal of active sealing, is examined. Also known as "viscoseals" screw seal elements have screw threads on the shaft or the housing. Threads formed by the screw profiles causes pumping action and generates sealing effect. The pressured fluid in the sealing system tends to flow towards the low pressure environment, but with the right direction of rotation and thread form, leaking fluid is moved back to the high pressured area. This design works as a volumetric pump.

\section{Experimental Set-Up}

In order to examine the effect of screw profile of seal elements and operating speeds on the sealing performance, the test system shown in Figure 1 is designed.

In order to drive the shaft in the system, an AC motor generating 1,2 $\mathrm{kW}$ is used. Motor speed can be adjusted between $0 \ldots 3600 \mathrm{rpm}$. The speed of the motor, can be adjusted with the speed regulator and monitored by the data acquisition software.

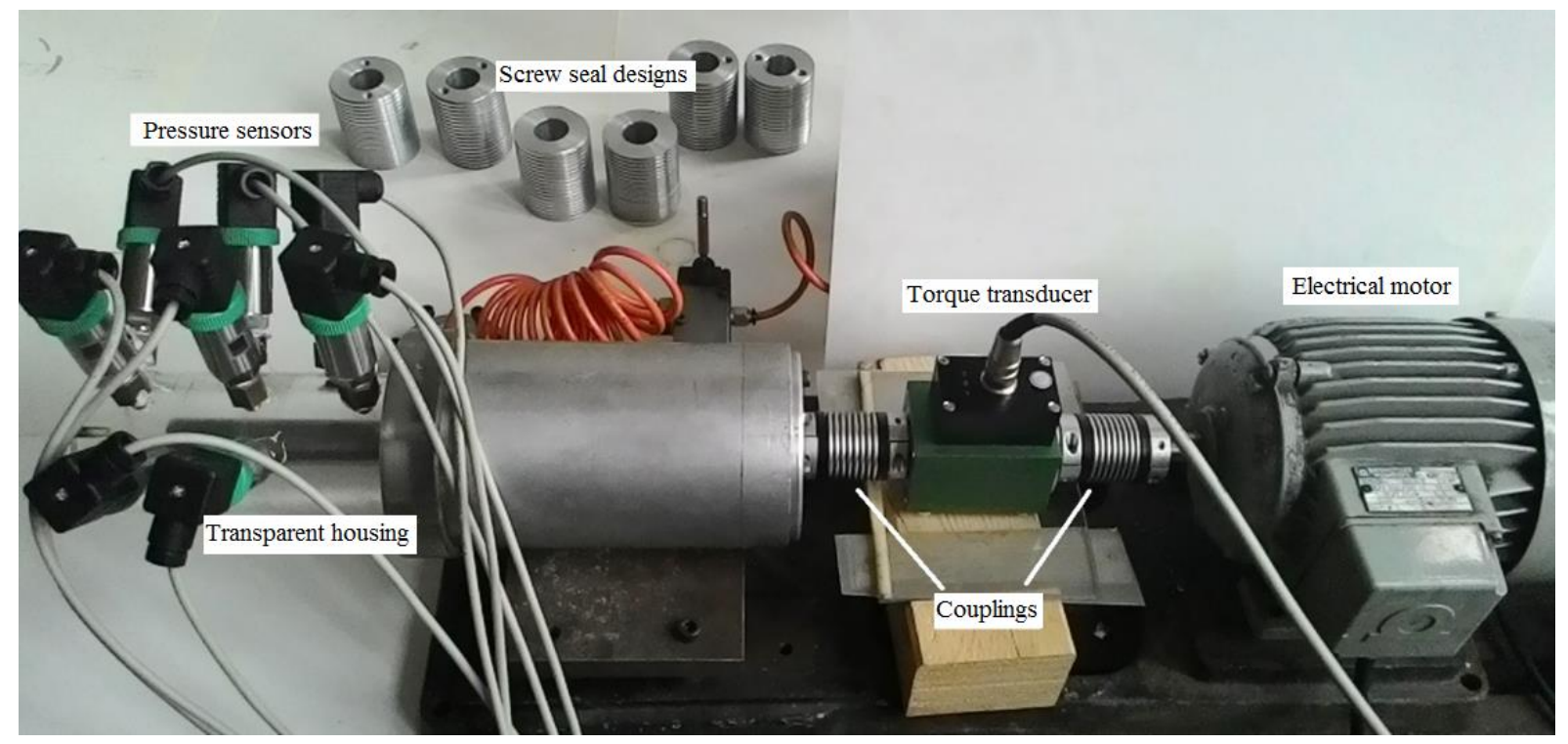

Fig. 1: Test system.

A torque transducer is placed between the motor and the shaft on which screw elements are mounted. Measuring range of Burster 8661 model torque transducer is $0 \ldots 10 \mathrm{Nm}$ 's. The output signal of device is between $0 \ldots 10 \mathrm{~V}$ 's and angle measurement with 1024 increments $/ 0.088^{\circ}$, can measure up to $6000 \mathrm{rpm}$. In order to obtain the pressure distribution of the sealing elements located inside the Plexiglas housing, pressure sensors are mounted on the housing. Sensors are Burster 8227 model, have measuring range of $0 \ldots 10$ bar and $0,25 \%$ of measurement accuracy. These sensors are suitable for liquid and gas pressure measurement, also can be used on static and dynamic applications. The data received from the sensors are transferred to the 16-channel data acquisition device of "IMC" brand Spartan-2 model.

On this test setup, experiments are carried out with $1200 \mathrm{rpm}, 2400 \mathrm{rpm}$ and $3600 \mathrm{rpm}$ rotational speeds. First experiments are carried out with air. Furthermore, in order to simulate actual operating conditions, some amount of oil is supplied to the system after system starts running. 


\section{3D Model of Screw Seal}

Screw seals are manufactured of 5000 series aluminium. For the ease of manufacturing the screw seals are designed as two components, a left-hand and a right-hand thread square profiled parts. The screw element used in these experiments has $2 \mathrm{~mm}$ of groove depth, $2 \mathrm{~mm}$ of groove width and $2 \mathrm{~mm}$ of tip width.
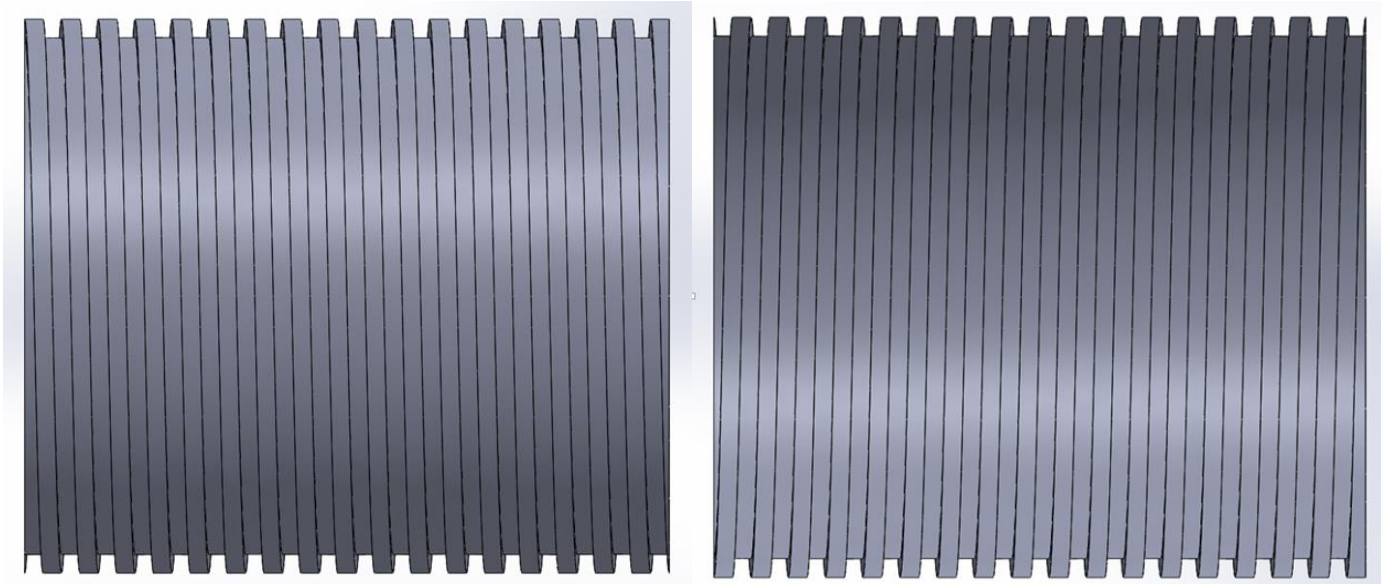

Fig. 2: 3D model of seal element's geometry.

The main factor of screw element effecting the sealing function can be assessed as the maximum pressure taken place the interface region of left-hand and right-hand screw elements (see Fig. 2). The fluids on each side cannot flow through the other side of the sealing elements because the pressure in both environments are lower than the pressure barrier in the interface. Higher pressure of interface than the each sides shows that sealing is obtained.

\section{Experimental Results}

In Figure 3, results of the experiments of the screw seals without any oil supply is shown. The experiments carried out at speeds of 1200, 2400 and $3600 \mathrm{rpm}$ respectively. The Figure 3 shows the pressure distribution along the screw seal elements. Sensor 5 gives the pressure formed on the interface of left and right hand screws. As seen from the Figure that rotational speed of screws affect pressure gradient on the system. When the rotational speed increases three times, corresponding pressure build-up also increases approximately 2.5 times. Average friction torques are measured for 1200, 2400 and $3600 \mathrm{rpm}$ speed as $0.15 \mathrm{Nm}, 0.17 \mathrm{Nm}$ and $0.25 \mathrm{Nm}$ respectively.

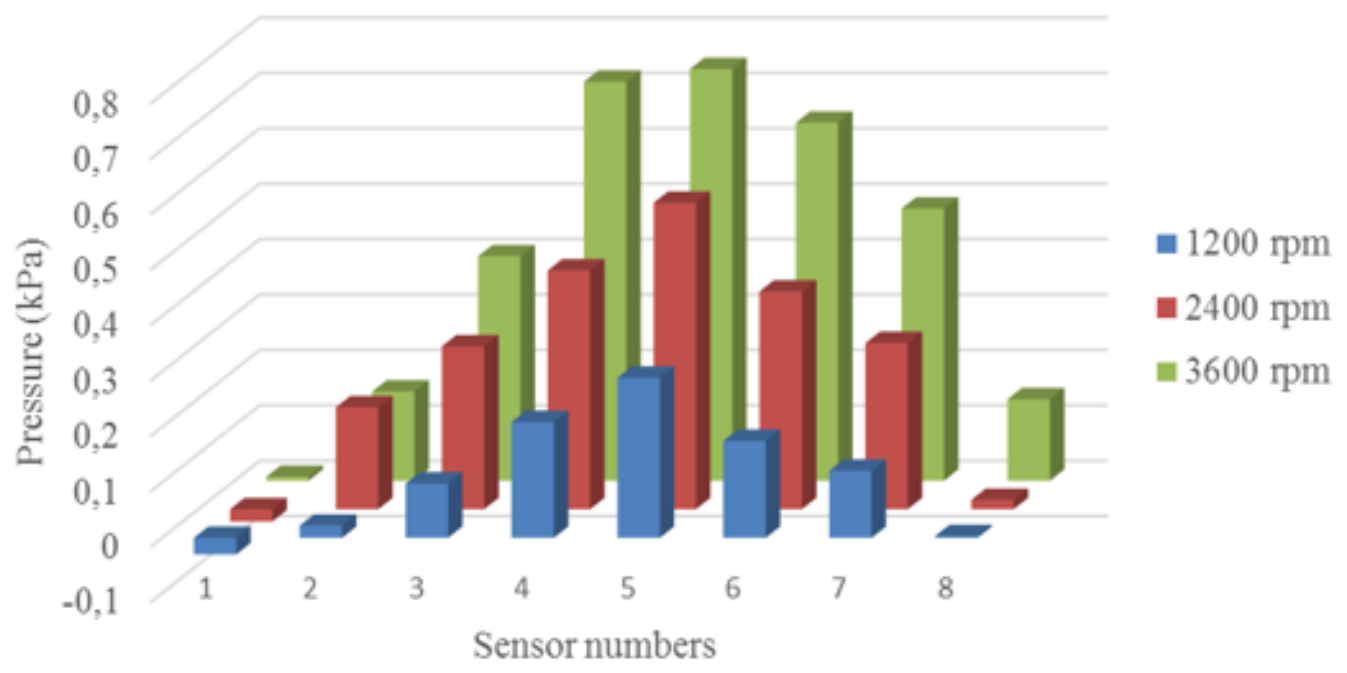

Fig. 3: The effect of rotational speed on the pressure distribution along the sealing elements. 
The industrial applications which this type of seals are used, cannot be considered as lubricant-free. In many cases oil particles or oil mist can be available on the systems. In order to simulate the actual operating conditions, some controlled amount of mineral oil is supplied to the system. During the experiments with mineral oil, 2 grams of Shell Dentax Gearbox Lube Oil is supplied on the screw seal area. Due to the fact that mineral oil is an uncompressible fluid, a relatively higher pressure gradient is observed around the interface of right and left screw seal parts. The form of oil barrier at the transition zone obtained in an experiments with the supply of mineral oil can be seen in Figure 4.

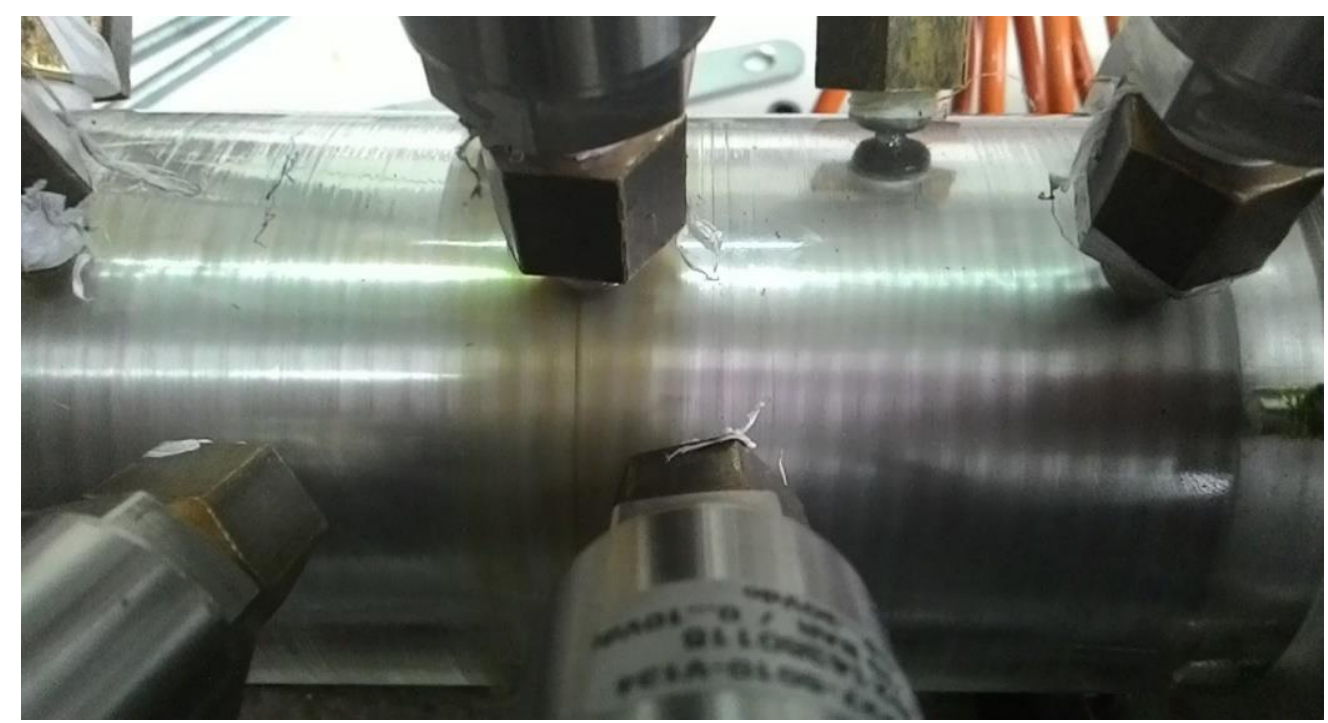

Fig. 4: Form of oil barrier at the transition area.

The experiments are repeated at the same conditions with supplying 2 grams of Shell Dentax Gearbox Lube Oil. In the Figure 5, only the pressures measured at the interface are given since other values are relatively low when compared to this pressure. When the rotational speed increases three times, corresponding pressure build-up increases approximately 1.75 times. This is due to the volumetric pump effect of seals with an uncompressible fluid. Average friction torques are measured for 1200, 2400 and $3600 \mathrm{rpm}$ speed as $0.57 \mathrm{Nm}, 0.68 \mathrm{Nm}$ and $0.84 \mathrm{Nm}$ respectively. The increase on torque values on oil supplied experiments with respect to oil-free ones are caused by relatively higher viscous resistance of oil.

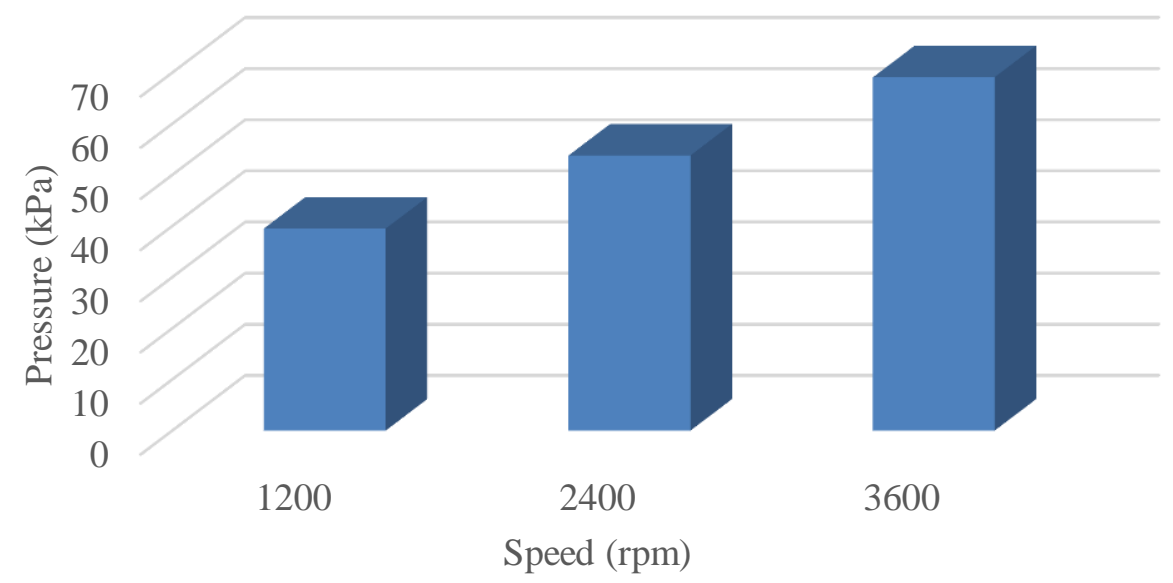

Fig. 5: Pressure values at the transition area. 


\section{Fluid Flow Analysis}

Using experimental data for validation of numerical flow analysis give rise to study more different geometries and to reach optimal design in a relatively short times. For that purpose, the geometry in the experiments is used in the fluid flow analysis. First of all, fluid domain is generated by using the solid body. Fluid, flows in the $20 \mu \mathrm{m}$ radial clearance between screw threads and housing. Since commercial grid generation programs have difficulties of controlling and generating computational grid in complex regions, a new code is used for mesh generating.

One of the most important condition is to obtain solutions that the model should be independent of mesh properties. For this purpose, coarse, medium and fine computational grids are generated. Mesh independency is achieved at 1.484.000 cells.

As the boundary conditions, two environments on each side of the screw seal elements are assigned as atmospheric pressure. Also no-slip boundary conditions are applied on wall boundaries of screw seal and housing. Rotational screw speed is set to $125 \mathrm{rad} / \mathrm{s}$.

K-eps RNG turbulence model is used for analysis since it gives better results. Second order discretization schemes for both temporal and special coordinates and 1e-4 convergence criteria are used in solutions. Furthermore, pressure and velocity values at two different positons in the fluid domain are monitored for convergence check.

Pressure distribution through the screw seal element with oil-free running is given on Figure 6 . Through the screw axis, pressure increases to maximum value of $0,04 \mathrm{kPa}$ around the interface.

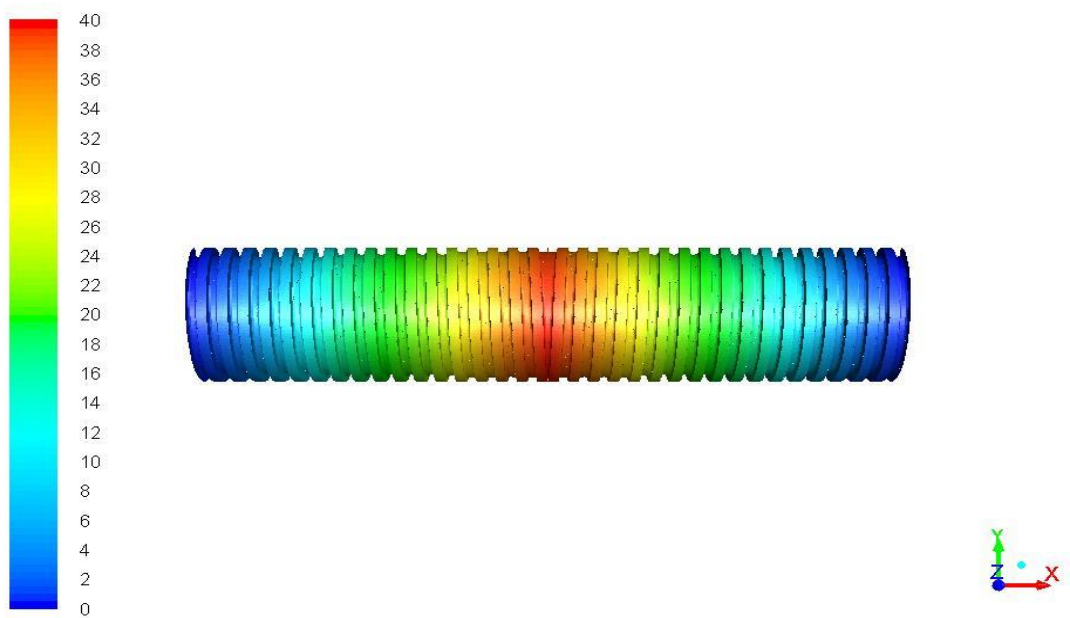

Fig. 6: Pressure distribution of the screw seal for oil-free running.

In Figure 7, pressure distribution around the transition zone in the middle of the screw seal is shown in details.

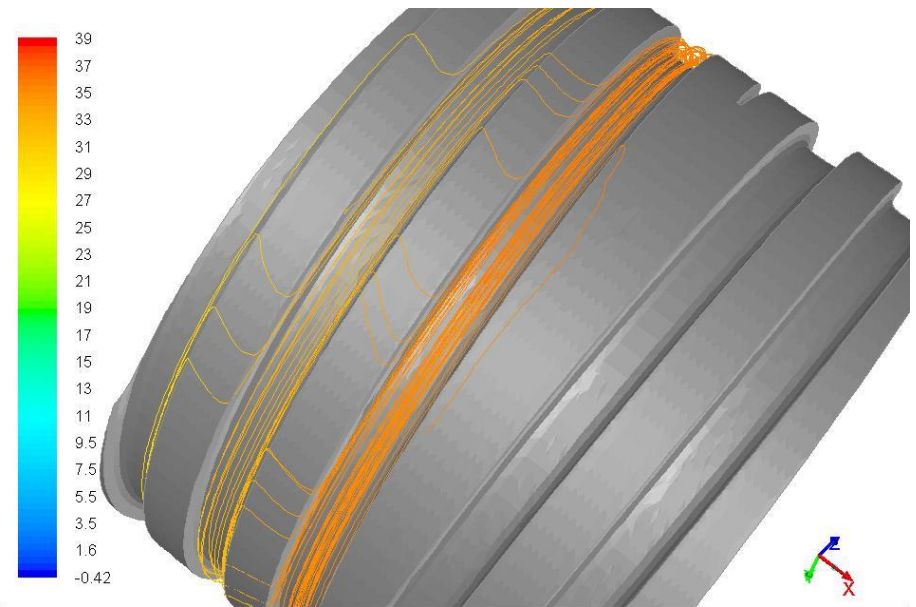

Fig. 7: Pressure distribution in the middle of the screw seal. 
In the analysis of oil supplied running conditions, the maximum pressure on the interface is increased to the value of $48 \mathrm{kPa}$ as seen in Fig. 8.

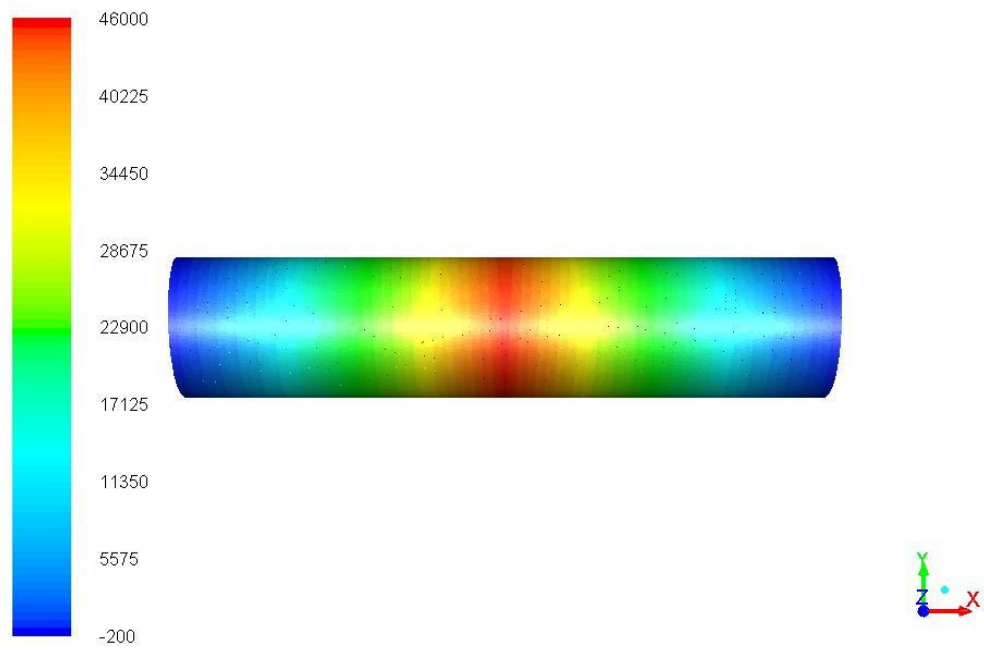

Fig. 8: Pressure distribution of screw seal with oil supplied running.

Comparing the experimental and numerical results, pressure values obtained at experiments for oil-free running are quite higher than numerical results. In numerical results, through the sealing element, a constant clearance, an ideal straight axis, and non-contacting screw-housing combination are defined. Therefore unfavourable effects resulting from the experimental setup do not taken into account in the analysis. Another issue is, that the interface of the screw seal has the optimal geometry in numerical analysis. Whereas screw seal is manufactured as two parts and assembled at the interface. The fluid (air) having low viscosity causes sensitivity to geometric deviations. Fluid leaking to the adjacent groove in the $30 \mu \mathrm{m}$ clearance, prevents the pressure increase as seen in Figure 7. The return leakage flows can be clearly seen from the Figure. In the experiments, this constant clearance cannot be obtained because of oscillation at high rotational speeds, furthermore in some experiments some amount of dry contact occurs between screw element and housing. After all, other than interface zone, the pressure values of experiments and analysis through the screw seal are compatible.

\section{Conclusion}

Regarding all these explanations, the following conclusions can be drawn from the present study:

- As seen both experimental and numerical studies, as the rotational speed increases, the pressure build-up at the interface also increases giving rise to better sealing performance.

- In oil-free running, friction torques resulting from the sealing elements are also affected from rotational speeds. However, this effect is not as evident as it is for pressure build-up.

- Numerical model gives consistent results with experimental data. However, numerical values for pressures in the interface for oil-free running are not close enough due to some experimental errors.

- Introducing oil to system causes the frictional torques to increase. The variation of friction torques with rotational speeds takes place in a relatively narrow range.

\section{Acknowledgements}

This research is a part of work for the project funded by Turkish National Research Council (TUBITAK) Research Fund: Grand No: 213M576 "Design of a New Contactless Screw Seal and Determining of Sealing Characteristics"

\section{References}

[1] L. P. Ludwig, T. N. Strom, and G. P. Allen, "Experimental Study of End Effect And Pressure Patterns In Helical Groove Fluid Film Seal (Viscoseal)," NASA Technical Note, TN D-3096 Lewis Research Center, Cleveland, OH, 1965. 
[2] D. W. Childs, "SSME Seal Test Program: Leakage Tests For Helically Grooved Seals," NASA Contract, NAS833716, Turbomachinery Laboratory, Texas A\&M Univ. College Station, Mech. Eng. Dept. Texas, TX.

[3] D. W. Childs, (1987) "SSME Seal Test Program: Test Results For Smooth, Hole Pattern and Helically-Grooved Stators” NASA Contract NAS8-35824, Turbomachinery Laboratory, Texas A\&M Univ. College Station, Mech. Eng. Dept. Texas, TX, 1983.

[4] J. Zuk, L. P. Ludwig, and R. L. Johnson, "Experimental and Theoretical Study of the Viscoseal," NASA Technical Memorandum, TM X-52245, Lewis Research Center, Cleveland, OH, 1966.

[5] K. E. Patterson, "An Analytical Model for the Prediction of Viscoseal Performance in the Regime from Continuum to Free-Molecule Flow," NASA Technical Note, N70-42191, the Univ. of Tennessee, Dept. of Mech. and Aerospace Eng. Knoxville, TN, 1970.

[6] L. P. Ludwig, T. N. Strom, and G. P. Allen, "Gas Ingestion and Sealing Capacity of Helical Groove Fluid Film Seal (Viscoseal) Using Sodium and Water as Sealed Fluids," NASA Technical Note, TN D-3348, Lewis Research Center, Cleveland, OH, 1966.

[7] R. J. G. Boon, "Observations on a Viscoseal in a Transparent Housing the Prevention of Leakage and Breakdown," MSc. thesis, Dept. Mech. and Aerospace Eng., Univ. of Tennessee, Knoxville, TN, 1969. 